Pacific

Journal of

Mathematics

TWISTED ALEXANDER POLYNOMIALS OF 2-BRIDGE KNOTS FOR PARABOLIC REPRESENTATIONS

TAKAYUKI MORIFUJI AND ANH T. TRAN

Volume 269 No. 2

June 2014 


\title{
TWISTED ALEXANDER POLYNOMIALS OF 2-BRIDGE KNOTS FOR PARABOLIC REPRESENTATIONS
}

\author{
TAKAYUKi MORIFUJI AND ANH T. TRAN
}

\begin{abstract}
In this paper we show that the twisted Alexander polynomial associated to a parabolic representation determines fiberedness and genus of a wide class of 2-bridge knots. As a corollary we give an affirmative answer to a conjecture of Dunfield, Friedl and Jackson for infinitely many hyperbolic knots.
\end{abstract}

\section{Introduction}

The twisted Alexander polynomial was introduced by Lin [2001] for knots in the 3 -sphere and by Wada [1994] for finitely presentable groups. It is a generalization of the classical Alexander polynomial and gives a powerful tool in low-dimensional topology. A theory of twisted Alexander polynomials has developed rapidly over the past ten years. One of the most important aspects is the determination of fiberedness [Friedl and Vidussi 2011b] and genus (the Thurston norm) [Friedl and Vidussi 2012] of knots by the collection of the twisted Alexander polynomials corresponding to all finite-dimensional representations. For literature on other applications and related topics, we refer to the survey paper [Friedl and Vidussi 2011a].

Let $K$ be a knot in $S^{3}$ and $G_{K}$ its knot group. Namely it is the fundamental group of the complement of $K$ in $S^{3}, G_{K}=\pi_{1}\left(S^{3} \backslash K\right)$. In this paper, we consider the twisted Alexander polynomial $\Delta_{K, \rho}(t) \in \mathbb{C}\left[t^{ \pm 1}\right]$ associated to a parabolic representation $\rho: G_{K} \rightarrow \mathrm{SL}_{2}(\mathbb{C})$. A typical example is the holonomy representation $\rho_{0}: G_{K} \rightarrow \mathrm{SL}_{2}(\mathbb{C})$ of a hyperbolic knot $K$, which is a lift of a discrete faithful representation $\bar{\rho}_{0}: G_{K} \rightarrow \mathrm{PSL}_{2}(\mathbb{C}) \cong \operatorname{Isom}^{+}\left(\mathbb{M}^{3}\right)$ such that $\mathbb{W}^{3} / \bar{\rho}_{0}\left(G_{K}\right) \cong S^{3} \backslash K$ where $\mathbb{H}^{3}$ denotes the upper half space model of hyperbolic 3-space (see [Thurston 1997]). Dunfield, Friedl and Jackson [Dunfield et al. 2012] numerically computed the twisted Alexander polynomial $\mathscr{T}_{K}(t)=\Delta_{K, \rho_{0}}(t)$, which is called the hyperbolic torsion polynomial, for all hyperbolic knots of 15 or fewer crossings. Based on these huge computations, they conjectured that the hyperbolic torsion polynomial determines the knot genus and, moreover, the knot is fibered if and only if $\mathscr{T}_{K}(t)$ is a monic polynomial. This conjecture is nice because it would imply the fiberedness and genus of a knot is determined by the twisted Alexander polynomial associated

MSC2010: 57M27.

Keywords: 2-bridge knot, parabolic representation, twisted Alexander polynomial. 
to a single representation. However it is widely open except for the hyperbolic twist knots [Morifuji 2012].

The purpose of this paper is to show that the above conjecture is true for a wide class of 2-bridge knots. Since 2-bridge knots are alternating, their fiberedness and genus can be determined by the Alexander polynomial [Crowell 1959; Murasugi 1958a; 1958b]. However there seems to be no a priori reason that the same must be true for the hyperbolic torsion polynomial.

For a prime $p$ and an integer $a$ between 1 and $p-1$, we say that $a$ is a primitive root modulo $p$ if it is a generator of the cyclic group $(\mathbb{Z} / p \mathbb{Z})^{*}$. Let $\mathscr{P}_{2}$ be the set of all odd primes $p$ such that 2 is a primitive root modulo $p$. Note that all primes $p=2 q+1$ such that $q$ is a prime $\equiv 1(\bmod 4)$ are contained in $\mathscr{P}_{2}$; see, for example, [LeVeque 1977, Theorem 5.6].

Theorem 1.1. Let $K$ be the knot $J(k, 2 n)$ as in Figure 1 , where $k>0$ and $n \in \mathbb{Z}$. For all hyperbolic knots $K$, the hyperbolic torsion polynomial $\mathscr{T}_{K}(t)$ determines the genus of $K$. Moreover for $k=2 m+1, k=2$ (twist knot), or $k=2 m$ and $|4 m n-1| \in \mathscr{P}_{2}$, the knot $J(k, 2 n)$ is fibered if and only if $\mathscr{T}_{K}(t)$ is monic.

As mentioned above, the holonomy representation $\rho_{0}$ is parabolic, so that Theorem 1.1 is an immediate corollary of the following theorem.

Theorem 1.2. Let $\rho: G_{K} \rightarrow \mathrm{SL}_{2}(\mathbb{C})$ be a parabolic representation of $K=J(k, 2 n)$. Then:

(1) $\Delta_{K, \rho}(t)$ determines the genus of $J(k, 2 n)$.

(2) $\Delta_{K, \rho}(t)$ determines the fiberedness of $J(k, 2 n)$ if $k=2 m+1, k=2$ (twist knot), or $k=2 m$ and $|4 m n-1| \in \mathscr{P}_{2}$.

Remark 1.3. (1) Suppose $k=2 m$ and $n>0$. Then $4 m n-1 \in \mathscr{P}_{2}$ if $4 m n-1$ is a prime and $2 m n-1$ is a prime $\equiv 1(\bmod 4)$.

(2) It is known that the conjugacy classes of parabolic representations into $\mathrm{SL}_{2}(\mathbb{C})$ of the knot $J(2 m, 2 n)$ can be described as the zero locus of an integral polynomial in one variable. The condition $|4 m n-1| \in \mathscr{P}_{2}$ in Theorem 1.2 (hence Theorem 1.1) assures the irreducibility over $\mathbb{Z}$ of this polynomial, see Section 5. We do not know whether Theorem 1.2(2) holds true for every integer $m$ and $n$.

This paper is organized as follows. In Section 2, we study nonabelian representations of the knot $J(k, 2 n)$ and give an explicit formula of the defining equation of the representation space. In Section 3, we investigate parabolic representations of $J(k, 2 n)$. In Section 4, we quickly review the definition of the twisted Alexander polynomial and some related work on fiberedness and genus of knots. In particular, we calculate the coefficients of the highest- and lowest-degree terms of the twisted Alexander polynomial associated to a nonabelian representation of $J(k, 2 n)$ and 


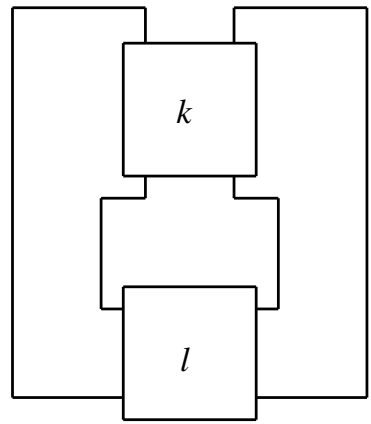

Figure 1. The knot $K=J(k, l)$. Here $k>0$ and $l=2 n(n \in \mathbb{Z})$ denote the numbers of half twists in each box. Positive numbers correspond to right-handed twists and negative numbers correspond to left-handed twists.

give the proof of Theorem 1.2(1). In Section 5, we discuss the fibering problem and prove Theorem 1.2(2).

\section{Non-abelian representations}

Let $K=J(k, l)$ be the knot as in Figure 1 . Note that $J(k, l)$ is a knot if and only if $k l$ is even, and is the trivial knot if $k l=0$. Furthermore, $J(k, l) \cong J(l, k)$ and $J(-k,-l)$ is the mirror image of $J(k, l)$. Hence, in the following, we consider $K=J(k, 2 n)$ for $k>0$ and $|n|>0$. When $k=2, J(2,2 n)$ is the twist knot.

In this section we explicitly calculate the defining equation of the nonabelian representation space of $J(k, 2 n)$.

By [Hoste and Shanahan 2004] the knot group of $K=J(k, 2 n)$ is presented by $G_{K}=\left\langle a, b \mid w^{n} a=b w^{n}\right\rangle$, where

$$
w= \begin{cases}\left(b a^{-1}\right)^{m}\left(b^{-1} a\right)^{m} & \text { for } k=2 m, \\ \left(b a^{-1}\right)^{m} b a\left(b^{-1} a\right)^{m} & \text { for } k=2 m+1 .\end{cases}
$$

Let $\left\{S_{i}(z)\right\}_{i}$ be the sequence of Chebyshev polynomials, defined by $S_{0}(z)=1$, $S_{1}(z)=z$, and $S_{i+1}(z)=z S_{i}(z)-S_{i-1}(z)$ for all positive integers $i$.

The following lemmas are standard; see, for example, [Tran 2013a, Lemma 2.4] and [Tran 2013b, Lemma 3.2].

Lemma 2.1. One has $S_{i}^{2}(z)-z S_{i}(z) S_{i-1}(z)+S_{i-1}^{2}(z)=1$.

Lemma 2.2. Suppose the sequence $\left\{M_{i}\right\}_{i}$ of $2 \times 2$ matrices satisfies the recurrence relation $M_{i+1}=z M_{i}-M_{i-1}$ for all integers $i$. Then

$$
\begin{aligned}
M_{i} & =S_{i-1}(z) M_{1}-S_{i-2}(z) M_{0}, \\
M_{i} & =S_{i}(z) M_{0}-S_{i-1}(z) M_{-1} .
\end{aligned}
$$


A representation $\rho: G_{K} \rightarrow \mathrm{SL}_{2}(\mathbb{C})$ is called nonabelian if $\rho\left(G_{K}\right)$ is a nonabelian subgroup of $\mathrm{SL}_{2}(\mathbb{C})$. Taking conjugation if necessary, we can assume that $\rho$ has the form

$$
\rho(a)=A=\left[\begin{array}{cc}
s & 1 \\
0 & s^{-1}
\end{array}\right] \quad \text { and } \quad \rho(b)=B=\left[\begin{array}{cc}
s & 0 \\
2-y & s^{-1}
\end{array}\right],
$$

where $(s, y) \in \mathbb{C}^{*} \times \mathbb{C}$ satisfies the matrix equation $W^{n} A-B W^{n}=0$. Here $W=\rho(w)$. It can be easily checked that $y=\operatorname{tr} A B^{-1}$ holds. Let $x=\operatorname{tr} A=\operatorname{tr} B=s+s^{-1}$.

Lemma 2.3. One has

$$
W A-B W=\left[\begin{array}{cc}
0 & \alpha_{k}(x, y) \\
(y-2) \alpha_{k}(x, y) & 0
\end{array}\right],
$$

where

$$
\alpha_{k}(x, y)= \begin{cases}1-\left(y+2-x^{2}\right) S_{m-1}(y)\left(S_{m-1}(y)-S_{m-2}(y)\right) & \text { for } k=2 m, \\ 1+\left(y+2-x^{2}\right) S_{m-1}(y)\left(S_{m}(y)-S_{m-1}(y)\right) & \text { for } k=2 m+1 .\end{cases}
$$

Proof. Recall that by the Cayley-Hamilton theorem, $M^{i+1}=(\operatorname{tr} M) M^{i}-M^{i-1}$ for all matrices $M \in \mathrm{SL}_{2}(\mathbb{C})$ and all integers $i$.

If $k=2 m$ then by applying (2-1) twice, we have

$$
\begin{aligned}
W A & =\left(B A^{-1}\right)^{m}\left(B^{-1} A\right)^{m} A \\
& =S_{m-1}^{2}(y) B A^{-1} B^{-1} A A-S_{m-1}(y) S_{m-2}(y)\left(B A^{-1} A+B^{-1} A A\right)+S_{m-2}^{2}(y) A .
\end{aligned}
$$

Similarly,

$$
\begin{aligned}
B W & =B\left(B A^{-1}\right)^{m}\left(B^{-1} A\right)^{m} \\
& =S_{m-1}^{2}(y) B B A^{-1} B^{-1} A-S_{m-1}(y) S_{m-2}(y)\left(B B A^{-1}+B B^{-1} A\right)+S_{m-2}^{2}(y) B .
\end{aligned}
$$

Hence, by direct calculations using (2-3), we obtain

$$
\begin{aligned}
W A-B W= & S_{m-1}^{2}(y)\left(B A^{-1} B^{-1} A A-B B A^{-1} B^{-1} A\right)+S_{m-2}^{2}(y)(A-B) \\
-S_{m-1}(y) S_{m-2}(y)\left(B A^{-1} A-B B A^{-1}+B^{-1} A A-B B^{-1} A\right) & \\
= & {\left[\begin{array}{cc}
0 & \alpha_{k}(x, y) \\
(y-2) \alpha_{k}(x, y) & 0
\end{array}\right], }
\end{aligned}
$$

where

$$
\alpha_{k}(x, y)=\left(s^{-2}+1+s^{2}-y\right) S_{m-1}^{2}(y)-\left(s^{-2}+s^{2}\right) S_{m-1}(y) S_{m-2}(y)+S_{m-2}^{2}(y) .
$$

Since $S_{m-1}^{2}(y)-y S_{m-1}(y) S_{m-2}(y)+S_{m-2}^{2}(y)=1$ (by Lemma 2.1) and $x=s+s^{-1}$,

$$
\alpha_{k}(x, y)=1-\left(y+2-x^{2}\right) S_{m-1}(y)\left(S_{m-1}(y)-S_{m-2}(y)\right) .
$$


If $k=2 m+1$ then by applying (2-2) twice, we have

$$
\begin{aligned}
W A= & \left(B A^{-1}\right)^{m} B A\left(B^{-1} A\right)^{m} A \\
= & S_{m}^{2}(y) B A A-S_{m}(y) S_{m-1}(y)\left(\left(B A^{-1}\right)^{-1} B A A+B A\left(B^{-1} A\right)^{-1} A\right) \\
& \quad+S_{m-1}^{2}(y)\left(B A^{-1}\right)^{-1} B A\left(B^{-1} A\right)^{-1} A \\
= & S_{m}^{2}(y) B A A-S_{m}(y) S_{m-1}(y)\left(A^{3}+B^{2} A\right)+S_{m-1}^{2}(y) A B A .
\end{aligned}
$$

Similarly,

$$
\begin{aligned}
B W= & B\left(B A^{-1}\right)^{m} B A\left(B^{-1} A\right)^{m} \\
= & S_{m}^{2}(y) B B A-S_{m}(y) S_{m-1}(y)\left(B\left(B A^{-1}\right)^{-1} B A+B B A\left(B^{-1} A\right)^{-1}\right) \\
& \quad+S_{m-1}^{2}(y) B\left(B A^{-1}\right)^{-1} B A\left(B^{-1} A\right)^{-1} \\
= & S_{m}^{2}(y) B B A-S_{m}(y) S_{m-1}(y)\left(B A^{2}+B^{3}\right)+S_{m-1}^{2}(y) B A B .
\end{aligned}
$$

Hence, by direct calculations using (2-3), we obtain

$$
\begin{aligned}
W A-B W & =S_{m}^{2}(y)(B A A-B B A)+S_{m-1}^{2}(y)(A B A-B A B) \\
& -S_{m}(y) S_{m-1}(y)\left(A^{3}-B A^{2}+B^{2} A-B^{2}\right) \\
& =\left[\begin{array}{cc}
0 & \alpha_{k}(x, y) \\
(y-2) \alpha_{k}(x, y) & 0
\end{array}\right],
\end{aligned}
$$

where

$$
\begin{aligned}
\alpha_{k}(x, y) & =S_{m}^{2}(y)-\left(s^{-2}+s^{2}\right) S_{m}(y) S_{m-1}(y)+\left(s^{-2}+1+s^{2}-y\right) S_{m-1}^{2}(y) \\
& =1+\left(y+2-x^{2}\right) S_{m-1}(y)\left(S_{m}(y)-S_{m-1}(y)\right) .
\end{aligned}
$$

This completes the proof of Lemma 2.3.

The proof of the following lemma is similar to that of Lemma 2.3.

Lemma 2.4. One has

$$
\operatorname{tr} W= \begin{cases}2+(y-2)\left(y+2-x^{2}\right) S_{m-1}^{2}(y) & \text { for } k=2 m, \\ x^{2}-y-(y-2)\left(y+2-x^{2}\right) S_{m}(y) S_{m-1}(y) & \text { for } k=2 m+1 .\end{cases}
$$

We are now ready to calculate the expression $W^{n} A-B W^{n}$ as follows. Let $\lambda=\operatorname{tr} W$.

Proposition 2.5. One has

$$
\begin{aligned}
& W^{n} A-B W^{n} \\
& =\left[\begin{array}{cc}
0 & S_{n-1}(\lambda) \alpha_{k}(x, y)-S_{n-2}(\lambda) \\
(y-2)\left(S_{n-1}(\lambda) \alpha_{k}(x, y)-S_{n-2}(\lambda)\right) & 0
\end{array}\right] .
\end{aligned}
$$


Proof. By applying (2-1) and Lemma 2.3, we have

$$
\begin{aligned}
W^{n} A-B W^{n} & =S_{n-1}(\lambda)(W A-B W)-S_{n-2}(\lambda)(A-B) \\
& =S_{n-1}(\lambda)\left[\begin{array}{cc}
0 & \alpha_{k}(x, y) \\
(y-2) \alpha_{k}(x, y) & 0
\end{array}\right]-S_{n-2}(\lambda)\left[\begin{array}{cc}
0 & 1 \\
y-2 & 0
\end{array}\right] .
\end{aligned}
$$

The proposition follows.

Proposition 2.5 implies that the assignment (2-3) gives a nonabelian representation $\rho: G_{K} \rightarrow \mathrm{SL}_{2}(\mathbb{C})$ if and only if $(s, y) \in \mathbb{C}^{*} \times \mathbb{C}$ satisfies the equation

$$
\phi_{k, 2 n}(x, y):=S_{n-1}(\lambda) \alpha_{k}(x, y)-S_{n-2}(\lambda)=0,
$$

where $\alpha_{k}(x, y)$ and $\lambda=\operatorname{tr} W$ are given by the formulas in Lemmas 2.3 and 2.4 respectively.

The polynomial $\phi_{k, 2 n}(x, y)$ is also known as the Riley polynomial [Riley 1984; Tkhang 1993] of $J(k, 2 n)$.

\section{Parabolic representations}

A representation $\rho: G_{K} \rightarrow \mathrm{SL}_{2}(\mathbb{C})$ is called parabolic if the meridian $\mu$ of $K$ is sent to a parabolic element (i.e., $\operatorname{tr} \rho(\mu)=2$ ) of $\mathrm{SL}_{2}(\mathbb{C})$ and $\rho\left(G_{K}\right)$ is nonabelian.

Let $K=J(k, 2 n)$. In this section we will show that if $\rho: G_{K} \rightarrow \mathrm{SL}_{2}(\mathbb{C})$ is a parabolic representation of the form

$$
\rho(a)=A=\left[\begin{array}{ll}
1 & 1 \\
0 & 1
\end{array}\right] \text { and } \rho(b)=B=\left[\begin{array}{cc}
1 & 0 \\
2-y & 1
\end{array}\right],
$$

where $y$ is a real number satisfying the equation $\phi_{k, 2 n}(2, y)=0$, then $y>2$.

Lemma 3.1. Suppose $x=2$. Then

$\alpha_{k}^{2}(x, y)-\alpha_{k}(x, y) \lambda+1= \begin{cases}(y-2)^{3} S_{m-1}^{4}(y) & \text { for } k=2 m, \\ (y-2)\left((y-2) S_{m}(y) S_{m-1}(y)+1\right)^{2} & \text { for } k=2 m+1 .\end{cases}$

Proof. If $k=2 m$ then $\alpha_{k}(x, y)=1-\left(y+2-x^{2}\right) S_{m-1}(y)\left(S_{m-1}(y)-S_{m-2}(y)\right)$ and $\lambda=2+(y-2)\left(y+2-x^{2}\right) S_{m-1}^{2}(y)$ by Lemmas 2.3 and 2.4. Hence, by direct calculations using $x=2$, we have

$\alpha_{k}^{2}(x, y)-\alpha_{k}(x, y) \lambda+1$

$=\left(-1-S_{m-1}^{2}(y)+y S_{m-1}^{2}(y)-y S_{m-1}(y) S_{m-2}(y)+S_{m-2}^{2}(y)\right)(y-2)^{2} S_{m-1}^{2}(y)$.

Since $S_{m-1}^{2}(y)-y S_{m-1}(y) S_{m-2}(y)+S_{m-2}^{2}(y)=1$ (by Lemma 2.1), we obtain

$$
\alpha_{k}^{2}(x, y)-\alpha_{k}(x, y) \lambda+1=(y-2)^{3} S_{m-1}^{4}(y) .
$$


If $k=2 m+1$ then $\alpha_{k}(x, y)=1+\left(y+2-x^{2}\right) S_{m-1}(y)\left(S_{m}(y)-S_{m-1}(y)\right)$ and $\lambda=x^{2}-y-(y-2)\left(y+2-x^{2}\right) S_{m}(y) S_{m-1}(y)$ by Lemmas 2.3 and 2.4. Hence, by direct calculations using $x=2$, we have

$$
\begin{aligned}
\alpha_{k}^{2}(x, y)-\alpha_{k}(x, y) \lambda+1=(y-2) & \left(1+(2-y) S_{m-1}^{2}(y)+(y-2) S_{m-1}^{4}(y)\right. \\
& +2(y-2) S_{m-1}(y) S_{m}(y)+(2-y) y S_{m-1}^{3}(y) S_{m}(y) \\
& \left.+\left(y^{2}-3 y+2\right) S_{m-1}^{2}(y) S_{m}^{2}(y)\right) .
\end{aligned}
$$

By replacing $y S_{m-1}^{3}(y) S_{m}(y)=S_{m-1}^{2}(y)\left(S_{m-1}^{2}(y)+S_{m}^{2}(y)-1\right)$ in this equality, we obtain

$$
\alpha_{k}^{2}(x, y)-\alpha_{k}(x, y) \lambda+1=(y-2)\left((y-2) S_{m}(y) S_{m-1}(y)+1\right)^{2},
$$

as claimed.

Proposition 3.2. Suppose $y$ is a real number satisfying the equation $\phi_{k, 2 n}(2, y)=0$. Then $y>2$.

Proof. Suppose $\phi_{k, 2 n}(x, y)=0$. Then $S_{n-1}(\lambda) \alpha_{k}(x, y)=S_{n-2}(\lambda)$. Hence

$$
\begin{aligned}
1 & =S_{n-1}^{2}(\lambda)-\lambda S_{n-1}(\lambda) S_{n-2}(\lambda)+S_{n-2}^{2}(\lambda) \\
& =\left(\alpha_{k}^{2}(x, y)-\alpha_{k}(x, y) \lambda+1\right) S_{n-1}^{2}(\lambda) .
\end{aligned}
$$

If we also suppose that $x=2$ and $y$ is a real number, then the above equality implies that $\alpha_{k}^{2}(x, y)-\alpha_{k}(x, y) \lambda+1>0$. By Lemma 3.1, we must have $y>2$.

\section{Twisted Alexander polynomials}

In this section we explicitly calculate the coefficients of the highest- and lowestdegree terms of the twisted Alexander polynomial associated to a nonabelian representation of $J(k, 2 n)$ and give the proof of Theorem 1.2(1).

Twisted Alexander polynomials. For a knot group $G_{K}=\pi_{1}\left(S^{3} \backslash K\right)$, we choose and fix a Wirtinger presentation

$$
G_{K}=\left\langle a_{1}, \ldots, a_{q} \mid r_{1}, \ldots, r_{q-1}\right\rangle .
$$

Then the abelianization homomorphism $f: G_{K} \rightarrow H_{1}\left(S^{3} \backslash K ; \mathbb{Z}\right) \cong \mathbb{Z}=\langle t\rangle$ is given by $f\left(a_{1}\right)=\cdots=f\left(a_{q}\right)=t$. Here we specify a generator $t$ of $H_{1}\left(S^{3} \backslash K ; \mathbb{Z}\right)$ and denote the sum in $\mathbb{Z}$ multiplicatively. Let us consider a linear representation $\rho: G_{K} \rightarrow \mathrm{SL}_{2}(\mathbb{C})$.

The maps $\rho$ and $f$ naturally induce two ring homomorphisms $\tilde{\rho}: \mathbb{Z}\left[G_{K}\right] \rightarrow$ $M(2, \mathbb{C})$ and $\tilde{f}: \mathbb{Z}\left[G_{K}\right] \rightarrow \mathbb{Z}\left[t^{ \pm 1}\right]$ respectively, where $\mathbb{Z}\left[G_{K}\right]$ is the group ring of $G_{K}$ and $M(2, \mathbb{C})$ is the matrix algebra of degree 2 over $\mathbb{C}$. Then $\tilde{\rho} \otimes \tilde{f}$ defines a ring homomorphism $\mathbb{Z}\left[G_{K}\right] \rightarrow M\left(2, \mathbb{C}\left[t^{ \pm 1}\right]\right)$. Let $F_{q}$ denote the free group 
on generators $a_{1}, \ldots, a_{q}$ and $\Phi: \mathbb{Z}\left[F_{q}\right] \rightarrow M\left(2, \mathbb{C}\left[t^{ \pm 1}\right]\right)$ the composition of the surjection $\mathbb{Z}\left[F_{q}\right] \rightarrow \mathbb{Z}\left[G_{K}\right]$ induced by the presentation of $G_{K}$ and the map $\tilde{\rho} \otimes \tilde{f}$ : $\mathbb{Z}\left[G_{K}\right] \rightarrow M\left(2, \mathbb{C}\left[t^{ \pm 1}\right]\right)$.

Consider the $(q-1) \times q$ matrix $M$ whose $(i, j)$-component is the $2 \times 2$ matrix

$$
\Phi\left(\frac{\partial r_{i}}{\partial a_{j}}\right) \in M\left(2, \mathbb{Z}\left[t^{ \pm 1}\right]\right),
$$

where $\partial / \partial a$ denotes the free differential calculus. For $1 \leq j \leq q$, let us denote by $M_{j}$ the $(q-1) \times(q-1)$ matrix obtained from $M$ by removing the $j$-th column. We regard $M_{j}$ as a $2(q-1) \times 2(q-1)$ matrix with coefficients in $\mathbb{C}\left[t^{ \pm 1}\right]$. Then Wada's twisted Alexander polynomial of a knot $K$ associated to a representation $\rho: G_{K} \rightarrow \mathrm{SL}_{2}(\mathbb{C})$ is defined to be a rational function

$$
\Delta_{K, \rho}(t)=\frac{\operatorname{det} M_{j}}{\operatorname{det} \Phi\left(1-a_{j}\right)}
$$

and moreover well-defined up to a factor $t^{2 n}(n \in \mathbb{Z})$. It is also known that if two representations $\rho, \rho^{\prime}$ are conjugate, then $\Delta_{K, \rho}(t)=\Delta_{K, \rho^{\prime}}(t)$ holds. See [Wada 1994] and [Goda et al. 2005] for details.

Remark 4.1. Let $\rho: G_{K} \rightarrow \mathrm{SL}_{2}(\mathbb{C})$ be a nonabelian representation.

(1) The twisted Alexander polynomial $\Delta_{K, \rho}(t)$ associated to $\rho$ is always a Laurent polynomial for any knot $K$ [Kitano and Morifuji 2005].

(2) The twisted Alexander polynomial is reciprocal; that is, $\Delta_{K, \rho}(t)=t^{i} \Delta_{K, \rho}\left(t^{-1}\right)$ for some $i \in \mathbb{Z}$ [Hillman et al. 2010; Friedl et al. 2012].

(3) If $K$ is a fibered knot, then $\Delta_{K, \rho}(t)$ is a monic polynomial for every nonabelian representation $\rho$ [Goda et al. 2005]. It is also known that the converse holds for alternating knots [Kim and Morifuji 2012, Remark 4.2].

(4) If $K$ is a knot of genus $g$, then $\operatorname{deg}\left(\Delta_{K, \rho}(t)\right) \leq 4 g-2$ [Friedl and Kim 2006]. Moreover if $K$ is fibered, then the equality holds [Kitano and Morifuji 2005].

We say the twisted Alexander polynomial $\Delta_{K, \rho}(t)$ determines the knot genus $g(K)$ if $\operatorname{deg}\left(\Delta_{K, \rho}(t)\right)=4 g(K)-2$ holds. For a hyperbolic knot $K$, the hyperbolic torsion polynomial $\mathscr{T}_{K}(t)$ is defined to be $\Delta_{K, \rho_{0}}(t)$ for the holonomy representation $\rho_{0}: G_{K} \rightarrow \mathrm{SL}_{2}(\mathbb{C})$. We note that it is normalized so that $\mathscr{T}_{K}(t)=\mathscr{T}_{K}\left(t^{-1}\right)$ holds.

Proof of part (1) of Theorem 1.2. It is known that the genus of $J(k, 2 n)$, where $k>1$ and $|n|>0$, is 1 if $k$ is even, and is $|n|$ if $k$ is odd. Moreover, the genus of $J(1,2 n)$ (the $(2,2 n-1)$-torus knot) is $n-1$ if $n>0$ and is $-n$ if $n<0$.

We first consider the case $n>0$. Let $r=w^{n} a w^{-n} b^{-1}$, where $w$ is as defined in Section 2. By direct calculations, we have

$$
\frac{\partial r}{\partial a}=w^{n}\left(1+(1-a)\left(w^{-1}+\cdots+w^{-n}\right) \frac{\partial w}{\partial a}\right),
$$


where, for $k=2 m$,

$$
\frac{\partial w}{\partial a}=-\left(b a^{-1}+\cdots+\left(b a^{-1}\right)^{m}\right)+\left(b a^{-1}\right)^{m}\left(1+b^{-1} a+\cdots+\left(b^{-1} a\right)^{m-1} b^{-1}\right),
$$

and, for $k=2 m+1$,

$$
\frac{\partial w}{\partial a}=-\left(b a^{-1}+\cdots+\left(b a^{-1}\right)^{m}\right)+\left(b a^{-1}\right)^{m} b\left(1+a b^{-1}+\cdots+\left(a b^{-1}\right)^{m}\right) .
$$

Suppose $\rho: G_{K} \rightarrow \mathrm{SL}_{2}(\mathbb{C})$ is a nonabelian representation given by (2-3). Then the twisted Alexander polynomial of $K$ associated to $\rho$ is

$$
\Delta_{K, \rho}(t)=\frac{\operatorname{det} \Phi\left(\frac{\partial r}{\partial a}\right)}{\operatorname{det} \Phi(1-b)}=\frac{\operatorname{det} \Phi\left(\frac{\partial r}{\partial a}\right)}{1-t x+t^{2}} .
$$

The case $J(2 m, 2 n), n>0$. From (4-1) we have

$$
\operatorname{det} \Phi\left(\frac{\partial r}{\partial a}\right)=\left|I+(I-t A)\left(W^{-1}+\cdots+W^{-n}\right) V\right|,
$$

where $I$ is the $2 \times 2$ identity matrix and

$$
V=-\left(B A^{-1}+\cdots+\left(B A^{-1}\right)^{m}\right)+\left(B A^{-1}\right)^{m}\left(I+B^{-1} A+\cdots+\left(B^{-1} A\right)^{m-1}\right) t^{-1} B^{-1} .
$$

The next lemma follows easily.

Lemma 4.2. The highest- and lowest-degree terms of $\operatorname{det} \Phi\left(\frac{\partial r}{\partial a}\right)$ are respectively

$$
\left|A\left(W^{-1}+\cdots+W^{-n}\right)\left(B A^{-1}+\cdots+\left(B A^{-1}\right)^{m}\right)\right| t^{2}
$$

and

$$
\left|\left(W^{-1}+\cdots+W^{-n}\right)\left(B A^{-1}\right)^{m}\left(I+B^{-1} A+\cdots+\left(B^{-1} A\right)^{m-1}\right) B^{-1}\right| t^{-2} .
$$

Let $\left\{T_{i}(z)\right\}_{i}$ be the sequence of Chebyshev polynomials defined by $T_{0}(z)=2$, $T_{1}(z)=z$ and $T_{i+1}(z)=z T_{i}(z)-T_{i-1}(z)$ for all integers $i$. Recall that $y=\operatorname{tr} A B^{-1}$ and $\lambda=\operatorname{tr} W$.

Proposition 4.3. The highest- and lowest-degree terms of $\operatorname{det} \Phi\left(\frac{\partial r}{\partial a}\right)$ are respectively

$$
\frac{T_{n}(\lambda)-2}{\lambda-2} \frac{T_{m}(y)-2}{y-2} t^{2} \text { and } \quad \frac{T_{n}(\lambda)-2}{\lambda-2} \frac{T_{m}(y)-2}{y-2} t^{-2} .
$$

Proof. Let $\beta_{ \pm}$be the roots of $z^{2}-\lambda z+1$ and $\gamma_{ \pm}$the roots of $z^{2}-y z+1$. Lemma 4.2 implies that the highest- and lowest degree terms of $\operatorname{det} \Phi(\partial r / \partial a)$ are respectively

$$
\begin{aligned}
\left(1+\beta_{+}+\cdots+\beta_{+}^{n-1}\right)\left(1+\beta_{-}+\right. & \left.\cdots+\beta_{-}^{n-1}\right) \\
& \times\left(1+\gamma_{+}+\cdots+\gamma_{+}^{m-1}\right)\left(1+\gamma_{-}+\cdots+\gamma_{-}^{m-1}\right) t^{2}
\end{aligned}
$$


and

$$
\begin{aligned}
\left(1+\beta_{+}+\cdots+\beta_{+}^{n-1}\right)\left(1+\beta_{-}\right. & \left.+\cdots+\beta_{-}^{n-1}\right) \\
& \times\left(1+\gamma_{+}+\cdots+\gamma_{+}^{m-1}\right)\left(1+\gamma_{-}+\cdots+\gamma_{-}^{m-1}\right) t^{-2} .
\end{aligned}
$$

Proposition 4.3 then follows from Lemma 4.4 below.

Lemma 4.4. $\left(1+\beta_{+}+\cdots+\beta_{+}^{n-1}\right)\left(1+\beta_{-}+\cdots+\beta_{-}^{n-1}\right)=\frac{T_{n}(\lambda)-2}{\lambda-2} \in \mathbb{Z}[\lambda]$.

Proof. The left-hand side is equal to

$$
\frac{\left(\beta_{+}^{n}-1\right)\left(\beta_{-}^{n}-1\right)}{\left(\beta_{+}-1\right)\left(\beta_{-}-1\right)}=\frac{\beta_{+}^{n}+\beta_{-}^{n}-2}{\beta_{+}+\beta_{-}-2}=\frac{T_{n}(\lambda)-2}{\lambda-2} .
$$

The lemma follows.

Proposition 4.3 implies that the highest- and lowest-degree terms of the twisted Alexander polynomial

$$
\Delta_{K, \rho}(t)=\frac{\operatorname{det} \Phi\left(\frac{\partial r}{\partial a}\right)}{1-t x+t^{2}}
$$

are respectively $U_{m, n}(y) t^{0}$ and $U_{m, n}(y) t^{-2}$, where

$$
U_{m, n}(y)=\frac{T_{n}(\lambda)-2}{\lambda-2} \frac{T_{m}(y)-2}{y-2} .
$$

Hence to prove Theorem 1.2(1) for $J(2 m, 2 n), n>0$, we only need to show that the coefficients of these terms are nonzero under the assumption that $\phi_{K}(2, y)=$ $S_{n-1}(\lambda) \alpha_{k}(2, y)-S_{n-2}(\lambda)=0$ (because the roots of this equation correspond to the parabolic representations). To this end, we show that at $x=2$ the polynomials $\phi_{K}(2, y)$ and $U_{m, n}(y)$ do not have any common zero $y \in \mathbb{C}$ (in fact, if they have a common zero, the highest- and lowest-degree terms vanish at $x=2$ ). It is equivalent to show that at $x=2$ these polynomials are relatively prime in $\mathbb{C}[y]$.

Recall that $\lambda=\operatorname{tr} W=(y-2)\left(y+2-x^{2}\right) S_{m-1}^{2}(y)+2$ and

$$
\alpha_{k}(x, y)=1-\left(y+2-x^{2}\right) S_{m-1}(y)\left(S_{m-1}(y)-S_{m-2}(y)\right) .
$$

The next two lemmas will complete the proof of Theorem 1.2(1) for $J(2 m, 2 n)$, $n>0$.

Lemma 4.5. Suppose $x=2$. Then $\operatorname{gcd}\left(\phi_{K}(x, y), \frac{T_{n}(\lambda)-2}{\lambda-2}\right)=1$ in $\mathbb{C}[y]$.

Proof. It is equivalent to show that at $x=2, \phi_{K}(x, y)$ and $\left(T_{n}(\lambda)-2\right) /(\lambda-2)$ do not have any common root $y \in \mathbb{C}$.

Suppose $T_{n}(\lambda)=2$ and $\lambda \neq 2$ then $\beta_{+}^{n}=\beta_{-}^{n}=1$ and $\beta_{+} \neq 1$. If $\beta_{+} \neq-1$ then $S_{n-1}(\lambda)=\left(\beta_{+}^{n}-\beta_{-}^{n}\right) /\left(\beta_{+}-\beta_{-}\right)=0$ and $S_{n-2}(\lambda)=\left(\beta_{+}^{n-1}-\beta_{-}^{n-1}\right) /\left(\beta_{+}-\beta_{-}\right)=-1$; hence $\phi_{K}(x, y)=1 \neq 0$. 
If $\beta_{+}=-1$ (in this case $n$ must be even) then $\lambda=-2$. It implies that $S_{n-1}(\lambda)=$ $-n$, and $S_{n-2}(\lambda)=n-1$. Hence $\phi_{K}(x, y)=-n \alpha_{k}(x, y)-(n-1)$.

Suppose $\phi_{K}(x, y)=0$. Then $\alpha_{k}(x, y)=1 / n-1$. We have

$$
\begin{aligned}
(y-2)\left(y+2-x^{2}\right) S_{m-1}^{2}(y) & =\lambda-2=-4, \\
\left(y+2-x^{2}\right)\left(S_{m-1}^{2}(y)-S_{m-1}(y) S_{m-2}(y)\right) & =1-\alpha_{k}(x, y)=2-\frac{1}{n} .
\end{aligned}
$$

Thus $\left(y+2-x^{2}\right) S_{m-1}^{2}(y)=\frac{-4}{y-2},\left(y+2-x^{2}\right) S_{m-1}(y) S_{m-2}(y)=-\left(\frac{4}{y-2}+2-\frac{1}{n}\right)$. Since

$$
S_{m-1}^{4}(y)-y S_{m-1}^{2}(y)\left(S_{m-1}(y) S_{m-2}(y)\right)+\left(S_{m-1}(y) S_{m-2}(y)\right)^{2}=S_{m-1}^{2}(y)
$$

(by Lemma 2.1), we must have

$$
\frac{16}{(y-2)^{2}}-\frac{4 y}{y-2}\left(\frac{4}{y-2}+2-\frac{1}{n}\right)+\left(\frac{4}{y-2}+2-\frac{1}{n}\right)^{2}=\frac{-4\left(y+2-x^{2}\right)}{y-2},
$$

that is, $y=2+4 n^{2} x^{2}$.

If $x \in \mathbb{R}$ then $y=2+4 n^{2} x^{2} \in \mathbb{R}$ and

$$
-4=(y-2)\left(y+2-x^{2}\right) S_{m-1}^{2}(y)=4 n^{2} x^{2}\left(4+\left(4 n^{2}-1\right) x^{2}\right) S_{m-1}^{2}(y) \geq 0,
$$

a contradiction. Hence $\phi_{K}(x, y) \neq 0$ when $\frac{T_{n}(\lambda)-2}{\lambda-2}=0$ and $x \in \mathbb{R}$. The lemma follows.

Lemma 4.6. Suppose $x=2$. Then $\operatorname{gcd}\left(\phi_{K}(x, y), \frac{T_{m}(y)-2}{y-2}\right)=1$ in $\mathbb{C}[y]$.

Proof. Suppose $T_{m}(y)=2$ and $y \neq 2$ then $\gamma_{+}^{m}=\gamma_{-}^{m}=1$ and $\gamma_{+} \neq 1$. If $\gamma_{+} \neq-1$ then $S_{m-1}(y)=\left(\gamma_{+}^{m}-\gamma_{-}^{m}\right) /\left(\gamma_{+}-\gamma_{-}\right)=0$, hence $\lambda=2$ and $\alpha_{k}(x, y)=1$. This implies that $\phi_{K}(x, y)=S_{n-1}(2)-S_{n-2}(2)=1 \neq 0$.

If $\gamma_{+}=-1$ (in this case $m$ must be even) then $y=-2$. We have

$$
\lambda=(y-2)\left(y+2-x^{2}\right) S_{m-1}^{2}(y)+2=4 m^{2} x^{2}+2
$$

and

$$
\alpha_{k}(x, y)=1-\left(y+2-x^{2}\right) S_{m-1}(y)\left(S_{m-1}(y)-S_{m-2}(y)\right)=m(2 m-1) x^{2}+1 .
$$

This implies that

$\phi_{K}(x, y)=S_{n-1}(\lambda) \alpha_{k}(x, y)-S_{n-2}(\lambda)=\left(m(2 m-1) x^{2}+1\right) S_{n-1}(\lambda)-S_{n-2}(\lambda)$.

If $x \in \mathbb{Z}$ then $\lambda=4 m^{2} x^{2}+2 \in \mathbb{Z}$ is even. This means that $\phi_{K}(x, y)$ is odd, since

$$
\phi_{K}(x, y) \equiv S_{n-1}(\lambda)-S_{n-2}(\lambda)(\bmod 2) .
$$

Thus $\phi_{K}(x, y) \neq 0$ when $\frac{T_{m}(y)-2}{y-2}=0$ and $x \in \mathbb{Z}$. The lemma follows. 
The case $J(2 m+1,2 n), n>0$. From (4-1) we have

$$
\operatorname{det} \Phi\left(\frac{\partial r}{\partial a}\right)=\left|I+(I-t A)\left(t^{-2} W^{-1}+\cdots+t^{-2 n} W^{-n}\right) V\right| t^{4 n},
$$

where

$$
V=-\left(B A^{-1}+\cdots+\left(B A^{-1}\right)^{m}\right)+t\left(B A^{-1}\right)^{m} B\left(I+A B^{-1}+\cdots+\left(A B^{-1}\right)^{m}\right) .
$$

We first consider the case $m=0$ (in this case we must have $n>1$ so that $K$ is a nontrivial knot). Then $W=B A$ and $\operatorname{det} \Phi\left(\frac{\partial r}{\partial a}\right)$

$$
\begin{aligned}
& =\left|I+(I-t A)\left(t^{-2} W^{-1}+\cdots+t^{-2 n} W^{-n}\right) t B\right| t^{4 n} \\
& =\left|\left(t^{-2} W^{-1}+\cdots+t^{-2 n} W^{-n}\right) t B-t A\left(t^{-4} W^{-2}+\cdots+t^{-2 n} W^{-n}\right) t B\right| t^{4 n} .
\end{aligned}
$$

This implies that the highest- and lowest-degree terms of det $\Phi(\partial r / \partial a)$ are $\left|t^{-1} W^{-1} B\right| t^{4 n}=t^{4 n-2}$ and $\left|t^{1-2 n} W^{-n} B\right| t^{4 n}=t^{2}$, respectively. Hence the highestand lowest degree terms of $\Delta_{K, \rho}(t)$ are $t^{4 n-4}$ and $t^{2}$, respectively. Since the genus of $J(1,2 n)$, where $n>1$, is $n-1$, we complete the proof of Theorem 1.2(1) for $J(1,2 n), n>1$.

We now consider the case $m>0$. In this case, we have the following.

Lemma 4.7. (1) The highest-degree term of $\operatorname{det} \Phi\left(\frac{\partial r}{\partial a}\right)$ is

$$
\begin{aligned}
\mid I-A W^{-1}\left(B A^{-1}\right)^{m} B\left(I+A B^{-1}+\cdots+\right. & \left.\left(A B^{-1}\right)^{m}\right) \mid t^{4 n} \\
& =\left|I+B A^{-1}+\cdots+\left(B A^{-1}\right)^{m-1}\right| t^{4 n} .
\end{aligned}
$$

(2) The lowest-degree term of $\operatorname{det} \Phi\left(\frac{\partial r}{\partial a}\right)$ is

$$
\left|-W^{-n}\left(B A^{-1}+\cdots+\left(B A^{-1}\right)^{m}\right)\right| t^{0}=\left|I+B A^{-1}+\cdots+\left(B A^{-1}\right)^{m-1}\right| t^{0} .
$$

Lemmas 4.7 and 4.4 imply the following.

Proposition 4.8. The highest- and lowest-degree terms of $\operatorname{det} \Phi\left(\frac{\partial r}{\partial a}\right)$ are respectively

$$
\frac{T_{m}(y)-2}{y-2} t^{4 n} \text { and } \quad \frac{T_{m}(y)-2}{y-2} t^{0} .
$$

Proposition 4.8 implies that the highest- and lowest-degree terms of $\Delta_{K, \rho}(t)$ are $\left(T_{m}(y)-2\right) /(y-2) t^{4 n-2}$ and $\left(T_{m}(y)-2\right) /(y-2) t^{0}$, respectively. Hence to prove Theorem 1.2(1) for $J(2 m+1,2 n)$, where $m, n>0$, we only need to show that at $x=2$ (parabolic representation) the polynomials $\phi_{K}(x, y)=S_{n-1}(\lambda) \alpha_{k}(x, y)-S_{n-2}(\lambda)$ and $\left(T_{m}(y)-2\right) /(y-2)$ are relatively prime in $\mathbb{C}[y]$. 
Recall that $\lambda=\operatorname{tr} W=x^{2}-y-(y-2)\left(y+2-x^{2}\right) S_{m}(y) S_{m-1}(y)$ and

$$
\alpha_{k}(x, y)=1+\left(y+2-x^{2}\right) S_{m-1}(y)\left(S_{m}(y)-S_{m-1}(y)\right) .
$$

The next lemma will complete the proof of Theorem 1.2(1) for $J(2 m+1,2 n)$, where $m, n>0$.

Lemma 4.9. Suppose $x=2$. Then $\operatorname{gcd}\left(\phi_{K}(x, y), \frac{T_{m}(y)-2}{y-2}\right)=1$ in $\mathbb{C}[y]$.

Proof. Suppose $T_{m}(y)=2$ and $y \neq 2$, then $\gamma_{+}^{m}=\gamma_{-}^{m}=1$ and $\gamma_{+} \neq 1$. If $\gamma_{+} \neq-1$ then $S_{m-1}(y)=0$ and $S_{m}(y)=1$; hence $\lambda=x^{2}-y$ and $\alpha_{k}(x, y)=1$. This implies that $\phi_{K}(x, y)=S_{n-1}(\lambda)-S_{n-2}(\lambda)$.

Since $\gamma_{+}^{m}=1$, we have $y=\gamma_{+}+\gamma_{+}^{-1}=2 \cos (2 \pi j / m)$ for some $0<j<m$. If $\phi_{K}(x, y)=S_{n-1}(\lambda)-S_{n-2}(\lambda)=0$ then $\lambda=2 \cos \left(\left(2 j^{\prime}-1\right) \pi /(2 n-1)\right)$ for some $1 \leq j^{\prime} \leq n-1$; see [Le and Tran 2012, Lemma 4.13], for example. Hence

$$
x^{2}=y+\lambda=2\left(\cos \frac{2 \pi j}{m}+\cos \frac{\left(2 j^{\prime}-1\right) \pi}{2 n-1}\right)<4 .
$$

If $\gamma_{+}=-1$ (in this case $m$ must be even) then $y=-2$. We have

$$
\lambda=-(y-2)\left(y+2-x^{2}\right) S_{m}(y) S_{m-1}(y)+x^{2}-y=(2 m+1)^{2} x^{2}+2
$$

and

$$
\alpha_{k}(x, y)=1+\left(y+2-x^{2}\right) S_{m-1}(y)\left(S_{m}(y)-S_{m-1}(y)\right)=m(2 m+1) x^{2}+1 .
$$

If $x$ is an even integer then $\lambda=(2 m+1)^{2} x^{2}+2$ is an even integer and $\alpha_{k}(x, y)=$ $m(2 m+1) x^{2}+1$ is an odd integer. Hence

$$
\phi_{K}(x, y)=S_{n-1}(\lambda) \alpha_{k}(x, y)-S_{n-2}(\lambda) \equiv S_{n-1}(\lambda)-S_{n-2}(\lambda)(\bmod 2)
$$

is odd and so is nonzero.

In both cases, we obtain $\phi_{K}(x, y) \neq 0$ when $\frac{T_{m}(y)-2}{y-2}=0$ and $x$ is an even integer at least 2 . The lemma follows.

Next we consider the case $n<0$. We put $l=-n(l>0)$. For $r=w^{n} a w^{-n} b^{-1}=$ $w^{-l} a w^{l} b^{-1}$, we have

$$
\frac{\partial r}{\partial a}={\frac{\partial w^{-l}}{\partial a}}^{-l}+w^{-l}\left(1+a \frac{\partial w^{l}}{\partial a}\right)=w^{-l}\left(1-(1-a)\left(1+w+\cdots+w^{l-1}\right) \frac{\partial w}{\partial a}\right) .
$$

The case $J(2 m, 2 n), n<0$. From (4-2) we have

$$
\operatorname{det} \Phi\left(\frac{\partial r}{\partial a}\right)=\left|I-(I-t A)\left(I+W+\cdots+W^{l-1}\right) V\right|,
$$

where

$$
V=-\left(B A^{-1}+\cdots+\left(B A^{-1}\right)^{m}\right)+\left(B A^{-1}\right)^{m}\left(I+B^{-1} A+\cdots+\left(B^{-1} A\right)^{m-1}\right) t^{-1} B^{-1} .
$$


Lemma 4.10. (1) The highest-degree term of $\operatorname{det} \Phi\left(\frac{\partial r}{\partial a}\right)$ is

$$
\left|-A\left(I+W+\cdots+W^{l-1}\right)\left(B A^{-1}+\cdots+\left(B A^{-1}\right)^{m}\right)\right| t^{2} .
$$

(2) The lowest-degree term of $\operatorname{det} \Phi\left(\frac{\partial r}{\partial a}\right)$ is

$$
\left|-\left(I+W+\cdots+W^{l-1}\right)\left(B A^{-1}\right)^{m}\left(I+B^{-1} A+\cdots+\left(B^{-1} A\right)^{m-1}\right) B^{-1}\right| t^{-2} .
$$

We can apply a similar argument to that of the parallel case with $n>0$ (page 441) to conclude that $\Delta_{K, \rho}(t)$, for $\rho$ parabolic, determines the knot genus in this case. The case $J(2 m+1,2 n), n<0$. From (4-2) we have

$$
\begin{aligned}
\operatorname{det} \Phi\left(\frac{\partial r}{\partial a}\right) & =\left|t^{-2 l} W^{-l}\left(I-(I-t A)\left(I+t^{2} W+\cdots+t^{2(l-1)} W^{l-1}\right) V\right)\right| \\
& =\left|I-(I-t A)\left(I+t^{2} W+\cdots+t^{2(l-1)} W^{l-1}\right) V\right| t^{-4 l},
\end{aligned}
$$

where

$$
V=-\left(B A^{-1}+\cdots+\left(B A^{-1}\right)^{m}\right)+t\left(B A^{-1}\right)^{m} B\left(I+A B^{-1}+\cdots+\left(A B^{-1}\right)^{m}\right) .
$$

Lemma 4.11. (1) The highest-degree term of $\operatorname{det} \Phi\left(\frac{\partial r}{\partial a}\right)$ is

$$
\begin{aligned}
\mid A W^{l-1}\left(B A^{-1}\right)^{m} B\left(I+A B^{-1}+\cdots+\left(A B^{-1}\right)^{m}\right) & \mid t^{0} \\
& =\left|I+A B^{-1}+\cdots+\left(A B^{-1}\right)^{m}\right| t^{0} .
\end{aligned}
$$

(2) The lowest-degree term of $\operatorname{det} \Phi\left(\frac{\partial r}{\partial a}\right)$ is

$$
\left|I+B A^{-1}+\cdots+\left(B A^{-1}\right)^{m}\right| t^{-4 l} .
$$

We can apply a similar argument to that of the parallel case with $n>0$ (page 444) to conclude again that $\Delta_{K, \rho}(t)$, for $\rho$ parabolic, determines the knot genus in this case.

The case analysis starting on page 441 covers all possibilities. Theorem 1.2(1) follows immediately.

\section{The fibering problem}

In this section we study some properties of the parabolic representation spaces of 2-bridge knots and give the proof of Theorem 1.2(2).

Parabolic representations of 2-bridge knots. Consider the 2-bridge knot $K=$ $\mathfrak{b}(p, q)$, where $p>q \geq 1$ are relatively prime. The knot group $G_{K}$ has a presentation $G_{K}=\langle a, b \mid w a=b w\rangle$, where $w=a^{\varepsilon_{1}} b^{\varepsilon_{2}} \cdots a^{\varepsilon_{p-2}} b^{\varepsilon_{p-1}}$ and $\varepsilon_{j}=(-1)^{\lfloor j q / p\rfloor}$ (see, e.g., [Burde and Zieschang 2003]). 
Let $\phi_{K}(x, y)$ be the defining equation for the nonabelian representations into $\mathrm{SL}_{2}(\mathbb{C})$ of $G_{K}$, where $x=\operatorname{tr} \rho(a)=\operatorname{tr} \rho(b)$ and $y=\operatorname{tr} \rho\left(a b^{-1}\right)$. Then $\phi_{K}(2, y)$ is the defining equation for the parabolic representations. It is known that $\phi_{K}(2, y) \in \mathbb{Z}[y]$ is a monic polynomial of degree $d=(p-1) / 2$; see [Riley 1984; Tkhang 1993].

We want to study the irreducibility of $\phi_{K}(2, y) \in \mathbb{Z}[y]$.

Lemma 5.1. One has $\phi_{K}(2, y)=S_{d}(y)+S_{d-1}(y)$ in $\mathbb{Z}_{2}[y]$.

Proof. The proof is similar to that of [Le and Tran 2012, Proposition A.2].

Suppose $\rho$ is a parabolic representation. Let $A=\rho(a), B=\rho(b)$ and $W=\rho(w)$. Taking conjugation if necessary, we can assume that

$$
A=\left[\begin{array}{ll}
1 & 1 \\
0 & 1
\end{array}\right] \text { and } B=\left[\begin{array}{cc}
1 & 0 \\
2-y & 1
\end{array}\right]
$$

where $y=\operatorname{tr} A B^{-1} \in \mathbb{C}$ satisfies the matrix equation $W A-B W=0$.

By the Cayley-Hamilton theorem applying for matrices in $\mathrm{SL}_{2}(\mathbb{C})$ we have $A+A^{-1}=\operatorname{tr}(A) I=2 I=0(\bmod 2)$, that is, $A^{-1}=A(\bmod 2)$. Similarly, $B^{-1}=$ $B(\bmod 2)$. This implies that $W=A^{\varepsilon_{1}} B^{\varepsilon_{2}} \ldots A^{\varepsilon_{2 d-1}} B^{\varepsilon_{2 d}}=(A B)^{d}(\bmod 2)$. By applying (2-2), we have

$$
\begin{aligned}
W A+B W & =(A B)^{d} A+B(A B)^{d} \\
& =S_{d}(y)(A+B)+S_{d-1}(y)\left(B^{-1}+A^{-1}\right) \\
& =\left(S_{d}(y)+S_{d-1}(y)\right)(A+B)(\bmod 2),
\end{aligned}
$$

where $A+B=\left[\begin{array}{ll}0 & 1 \\ y & 0\end{array}\right](\bmod 2)$. Hence $\phi_{K}(2, y)=S_{d}(y)+S_{d-1}(y)$ in $\mathbb{Z}_{2}[y]$.

Recall from the Introduction that $\mathscr{P}_{2}$ is the set of all odd primes $p$ such that 2 is a primitive root modulo $p$.

Lemma 5.2. Suppose $p \in \mathscr{P}_{2}$. Then $S_{d}(y)+S_{d-1}(y) \in \mathbb{Z}_{2}[y]$ is irreducible.

Proof. Let $y=u+u^{-1}$. Then

$$
S_{d}(y)+S_{d-1}(y)=\frac{u^{d+1}+u^{-(d+1)}}{u+u^{-1}}+\frac{u^{d}+u^{-d}}{u+u^{-1}}=u^{-d} \frac{1+u^{2 d+1}}{1+u} .
$$

Suppose $p \in \mathscr{P}_{2}$. We will show that $\left(1+u^{p}\right) /(1+u) \in \mathbb{Z}_{2}[u]$ is irreducible. This will imply that $S_{d}(y)+S_{d-1}(y) \in \mathbb{Z}_{2}[y]$ is irreducible.

We have $\left(1+u^{p}\right) /(1+u)=u^{p-1}+\cdots+u+1$ is the $p$-th-cyclotomic polynomial $C_{p}(u) \in \mathbb{Z}_{2}[u]$ (since $p$ is an odd prime). It is well known that $C_{p}(u) \in \mathbb{Z}_{2}[u]$ is irreducible if $p \in \mathscr{P}_{2}$; see for example, [Roman 2006, Theorem 11.2.8]. The lemma follows.

Proposition 5.3. Suppose $p \in \mathscr{P}_{2}$. Then $\phi_{K}(2, y) \in \mathbb{Z}[y]$ is irreducible. 
Proof. By Lemma 5.1, $\phi_{K}(2, y)=S_{d}(y)+S_{d-1}(y) \in \mathbb{Z}_{2}[y]$. Since $p \in \mathscr{P}_{2}$, the polynomial $S_{d}(y)+S_{d-1}(y) \in \mathbb{Z}_{2}[y]$ is irreducible by Lemma 5.2. This implies that $\phi_{K}(2, y)$ is irreducible in $\mathbb{Z}_{2}[y]$. Since $\phi_{K}(2, y) \in \mathbb{Z}[y]$ is a monic polynomial in $y$, it is irreducible in $\mathbb{Z}[y]$.

Proof of part (2) of Theorem 1.2. It is known that $J(k, 2 n)$ is fibered only for the trivial knot $J(k, 0)$, the trefoil knot $J(2,2)$, the figure eight knot $J(2,-2)$, the knots $J(1,2 n)$ for any $n$, and the knots $J(3,2 n)$ for $n>0$.

The case $J(2 m, 2 n), m>1$. We will apply Proposition 5.3 to study the fibering problem for $K=J(2 m, 2 n)$.

Let $p=|4 m n-1|$ then it is known that $\phi_{K}(2, y)$ has degree $(p-1) / 2$. By Proposition 5.3, the polynomial $\phi_{K}(2, y) \in \mathbb{Z}[y]$ is irreducible if $p \in \mathscr{P}_{2}$.

Proposition 5.4. Suppose $m>1$ and $p=|4 m n-1| \in \mathscr{P}_{2}$. Then $\Delta_{K, \rho}(t)$ is nonmonic for every parabolic representation $\rho$.

Proof. We only need to consider the case $n>0$. The case $n<0$ is similar.

Suppose $\rho$ is a parabolic representation, that is, $x=2$. Since $k=2 m$, by Proposition 4.3 the coefficient of the highest-degree term of $\Delta_{K, \rho}(t)$ is $h(y)=$ $\left(T_{n}(\lambda)-2\right) /(\lambda-2) \times\left(T_{m}(y)-2\right) /(y-2)$, an integer polynomial in $y$ of degree $(n-1)(2 m)+(m-1)=2 m n-(m+1)<2 m n-1=(p-1) / 2$.

Since $p \in \mathscr{P}_{2}$, the polynomial $\phi_{K}(2, y) \in \mathbb{Z}[y]$ is irreducible. This implies that $\phi_{K}(2, y)$ does not divide $h(y)-1$ in $\mathbb{Z}[y]$. Hence $h(y) \neq 1$ when $\phi_{K}(2, y)=0$. The proposition follows.

Twist knots $J(2,2 n)$. For $K=J(2,2 n)$ we have $\lambda=y^{2}-y x^{2}+2 x^{2}-2$ and $\left.\overline{\phi_{K}(x, y)=-(y+1}-x^{2}\right) S_{n-1}(\lambda)-S_{n-2}(\lambda)$. Suppose $\rho$ is a nonabelian representation. By Proposition 4.3 the coefficient of the highest-degree term of $\Delta_{K, \rho}(t)$ is $\left(T_{n}(\lambda)-2\right) /(\lambda-2)$. We want to show that for $|n|>1$, we have $\left(T_{n}(\lambda)-2\right) /(\lambda-2) \neq 1$ when $\phi_{K}(x, y)=0$ and $x=2$. This will imply that for any parabolic representation $\rho, \Delta_{K, \rho}(t)$ is monic if and only if $|n|=1$.

Lemma 5.5. If $x=2$, then $\operatorname{gcd}\left(\phi_{K}(2, y), \frac{T_{n}(\lambda)-2}{\lambda-2}-1\right)=1$ in $\mathbb{C}[y]$ for $|n|>1$.

Proof. We only need to consider the case $n>1$. The case $n<-1$ is similar.

Suppose $T_{n}(\lambda)=\lambda$ and $\lambda \neq 2$. Then $\beta_{+}^{n}+\beta_{-}^{n}=\beta_{+}+\beta_{-}$, i.e., $\beta_{+}^{n-1}=1$ or $\beta_{+}^{n+1}=1$. It implies that $\lambda=-2$, or $\lambda=2 \cos 2 j \pi /(n-1)$ for some $1 \leq j \leq n-2$ and $j \neq(n-1) / 2$, or $\lambda=2 \cos 2 j \pi /(n+1)$ for some $1 \leq j \leq n$ and $j \neq(n+1) / 2$.

Case 1: $\lambda=-2$ (in this case $n$ must be odd). By similar arguments as in the proof of Lemma 4.5, we have $\phi_{K}(x, y) \neq 0$ if $x \in \mathbb{R}$.

Case $2: \lambda=2 \cos 2 j \pi /(n-1)$ for some $1 \leq j \leq n-2$ and $j \neq(n-1) / 2$. Then $S_{n-1}(\lambda)=1$ and $S_{n-2}(\lambda)=0$, hence $\phi_{K}(x, y)=-\left(y+1-x^{2}\right)$. 
Suppose $\phi_{K}(x, y)=0$. Then $y=x^{2}-1$ and $\lambda=y^{2}-y x^{2}+2 x^{2}-2=x^{2}-1$. This cannot occur if $x^{2}-1 \geq 2$, since $\lambda<2$. Hence $\phi_{K}(x, y) \neq 0$ if $x^{2} \geq 3$.

Case 3: $\lambda=2 \cos 2 j \pi /(n+1)$ for some $1 \leq j \leq n$ and $j \neq(n+1) / 2$. Then $S_{n-1}(\lambda)=-1$ and $S_{n-2}(\lambda)=-\lambda$, hence $\phi_{K}(x, y)=y+1-x^{2}+\lambda$.

Suppose $\phi_{K}(x, y)=0$. Then $y=x^{2}-\lambda-1$ and $\lambda=y^{2}-y x^{2}+2 x^{2}-2=$ $\lambda^{2}+\lambda\left(2-x^{2}\right)+x^{2}-1$, that is, $\lambda^{2}-\lambda\left(x^{2}-1\right)+x^{2}-1=0$. This equation does not have any real solution $\lambda$ if $1<x^{2}<5$. Hence $\phi_{K}(x, y) \neq 0$ if $1<x^{2}<5$.

In all cases, $\phi_{K}(x, y) \neq 0$ when $\left(T_{n}(\lambda)-2\right) /(\lambda-2)=1$ and $3 \leq x^{2}<5$. The lemma follows.

Remark 5.6. Lemma 5.5 gives a proof of [Morifuji 2012, Theorem 1.2] that does not use the irreducibility of $\phi_{J(2,2 n)}(2, y) \in \mathbb{Z}[y]$ proved in [Hoste and Shanahan 2001].

The case $J(2 m+1,2 n)$. Let $K=J(2 m+1,2 n)$. Suppose $\rho$ is a nonabelian representation. By Proposition 4.8 and Lemma 4.11, the coefficient of the highestdegree term of $\Delta_{K, \rho}(t)$ is

$$
\frac{T_{m}(y)-2}{y-2} \text { if } n>0 \quad \text { and } \quad \frac{T_{m+1}(y)-2}{y-2} \text { if } n<0 .
$$

We want to show that for $m>1$, we have $\left(T_{m}(y)-2\right) /(y-2) \neq 1$ when $\phi_{K}(2, y)=0$. This will imply that for any parabolic representation $\rho, \Delta_{K, \rho}(t)$ is monic if and only if $K=J(1,2 n)$, or $K=J(3,2 n)$ and $n>0$.

The key point of the proof of the following lemma is to apply Proposition 3.2.

Lemma 5.7. If $x=2$, then $\operatorname{gcd}\left(\phi_{K}(2, y), \frac{T_{m}(y)-2}{y-2}-1\right)=1$ in $\mathbb{C}[y]$ for $m>1$. Proof. Suppose $T_{m}(y)=y$ and $y \neq 2$. Then $\gamma_{+}^{m}+\gamma_{-}^{m}=\gamma_{+}+\gamma_{-}$, i.e., $\gamma_{+}^{m-1}=1$ or $\gamma_{+}^{m+1}=1$. This implies that $y=-2$, or $y=2 \cos 2 j \pi /(m-1)$ for some $1 \leq j \leq m-2$ and $j \neq(m-1) / 2$, or $y=2 \cos 2 j \pi /(m+1)$ for some $1 \leq j \leq m$ and $j \neq(m+1) / 2$. In all cases, $y \in \mathbb{R}$ and $y<2$. Proposition 3.2 then implies that $\phi_{K}(2, y) \neq 0$. The lemma follows.

The case analysis on the last two pages cover all possibilities, showing part (2) of Theorem 1.2. This completes the proof of the theorem.

\section{Acknowledgements}

Morifuji was partially supported by Grant-in-Aid for Scientific Research 23540076 from the Ministry of Education, Culture, Sports, Science and Technology, Japan. Tran thanks T. T. Q. Le for helpful discussions. Both authors would like to thank the referee for comments and suggestions. 


\section{References}

[Burde and Zieschang 2003] G. Burde and H. Zieschang, Knots, 2nd ed., de Gruyter Studies in Mathematics 5, de Gruyter, Berlin, 2003. MR 2003m:57005 Zbl 1009.57003

[Crowell 1959] R. Crowell, "Genus of alternating link types”, Ann. of Math. (2) 69 (1959), 258-275. MR 20 \#6103b Zbl 0111.35803

[Dunfield et al. 2012] N. M. Dunfield, S. Friedl, and N. Jackson, "Twisted Alexander polynomials of hyperbolic knots", Exp. Math. 21:4 (2012), 329-352. MR 3004250 Zbl 1266.57008

[Friedl and Kim 2006] S. Friedl and T. Kim, "The Thurston norm, fibered manifolds and twisted Alexander polynomials”, Topology 45:6 (2006), 929-953. MR 2007g:57020 Zbl 1105.57009

[Friedl and Vidussi 2011a] S. Friedl and S. Vidussi, "A survey of twisted Alexander polynomials", pp. 45-94 in The mathematics of knots, edited by M. Banagl and D. Vogel, Contrib. Math. Comput. Sci. 1, Springer, Heidelberg, 2011. MR 2012f:57024 Zbl 1223.57012

[Friedl and Vidussi 2011b] S. Friedl and S. Vidussi, "Twisted Alexander polynomials detect fibered 3-manifolds", Ann. of Math. (2) 173:3 (2011), 1587-1643. MR 2012f:57025 Zbl 1231.57020

[Friedl and Vidussi 2012] S. Friedl and S. Vidussi, "The Thurston norm and twisted Alexander polynomials", preprint, 2012. To appear in J. Reine Angew. Math. arXiv 1204.6456

[Friedl et al. 2012] S. Friedl, T. Kim, and T. Kitayama, "Poincaré duality and degrees of twisted Alexander polynomials", Indiana Univ. Math. J. 61:1 (2012), 147-192. MR 3029395 Zbl 1273.57009

[Goda et al. 2005] H. Goda, T. Kitano, and T. Morifuji, "Reidemeister torsion, twisted Alexander polynomial and fibered knots", Comment. Math. Helv. 80:1 (2005), 51-61. MR 2005m:57008 Zbl 1066.57008

[Hillman et al. 2010] J. A. Hillman, D. S. Silver, and S. G. Williams, "On reciprocality of twisted Alexander invariants”, Algebr. Geom. Topol. 10:2 (2010), 1017-1026. MR 2011j:57021 Zbl 1200. 57005

[Hoste and Shanahan 2001] J. Hoste and P. D. Shanahan, "Trace fields of twist knots", J. Knot Theory Ramifications 10:4 (2001), 625-639. MR 2002b:57012 Zbl 1003.57014

[Hoste and Shanahan 2004] J. Hoste and P. D. Shanahan, "A formula for the A-polynomial of twist knots”, J. Knot Theory Ramifications 13:2 (2004), 193-209. MR 2005c:57006 Zbl 1057.57010

[Kim and Morifuji 2012] T. Kim and T. Morifuji, "Twisted Alexander polynomials and character varieties of 2-bridge knot groups", Internat. J. Math. 23:6 (2012), 1250022, 24. MR 2925470 Zbl 1255.57013

[Kitano and Morifuji 2005] T. Kitano and T. Morifuji, "Divisibility of twisted Alexander polynomials and fibered knots", Ann. Sc. Norm. Super. Pisa Cl. Sci. (5) 4:1 (2005), 179-186. MR 2006e:57006 Zbl 1117.57004

[Le and Tran 2012] T. T. Q. Le and A. T. Tran, "On the AJ conjecture for knots", preprint, 2012. arXiv 1111.5258

[LeVeque 1977] W. J. LeVeque, Fundamentals of number theory, Addison-Wesley, Reading, MA, 1977. MR 58 \#465 Zbl 0368.10001

[Lin 2001] X. S. Lin, "Representations of knot groups and twisted Alexander polynomials", Acta Math. Sin. (Engl. Ser.) 17:3 (2001), 361-380. MR 2003f:57018 Zbl 0986.57003

[Morifuji 2012] T. Morifuji, "On a conjecture of Dunfield, Friedl and Jackson”, C. R. Math. Acad. Sci. Paris 350:19-20 (2012), 921-924. MR 2990904 Zbl 1253.57007

[Murasugi 1958a] K. Murasugi, "On the genus of the alternating knot, I", J. Math. Soc. Japan 10 (1958), 94-105. MR 20 \#6103a Zbl 0084.19301 
[Murasugi 1958b] K. Murasugi, "On the genus of the alternating knot, II", J. Math. Soc. Japan 10 (1958), 235-248. MR 20 \#6103a Zbl 0106.16701

[Riley 1984] R. Riley, "Nonabelian representations of 2-bridge knot groups", Quart. J. Math. Oxford Ser. (2) 35:138 (1984), 191-208. MR 85i:20043 Zbl 0549.57005

[Roman 2006] S. Roman, Field theory, 2nd ed., Graduate Texts in Mathematics 158, Springer, New York, 2006. MR 2006e:12001 Zbl 1172.12001

[Thurston 1997] W. P. Thurston, Three-dimensional geometry and topology, I, Princeton Mathematical Series 35, Princeton University Press, 1997. MR 97m:57016 Zbl 0873.57001

[Tkhang 1993] L. T. K. Tkhang, "Varieties of representations and their subvarieties of cohomology jumps for knot groups", Mat. Sb. 184:2 (1993), 57-82. In Russian; translated in Russ. Acad. Sci. Sb. Math. 78:1 (1994), 187-209. MR 94a:57016 Zbl 0836.57004

[Tran 2013a] A. T. Tran, "The universal character ring of some families of one-relator groups", Algebr. Geom. Topol. 13:4 (2013), 2317-2333. MR 3073918 Zbl 06185351

[Tran 2013b] A. T. Tran, “The universal character ring of the $(-2,2 m+1,2 n)$-pretzel link", Int. J. Math. 24:8 (2013), 1350063, 13. Zbl 06220065

[Wada 1994] M. Wada, “Twisted Alexander polynomial for finitely presentable groups", Topology 33:2 (1994), 241-256. MR 95g:57021 Zbl 0822.57006

Received December 21, 2012. Revised August 22, 2013.

TAKAYUKI MORIFUJI

DEPARTMENT OF MATHEMATICS

KEIO UNIVERSITY

HIYOSHI CAMPUS

YOKOHAMA 223-8521

JAPAN

morifuji@z8.keio.jp

ANH T. TRAN

DEPARTMENT OF MATHEMATICS

THE OHIO STATE UNIVERSITY

100 MATH TOWER

231 West 18Th AVEnUE

Columbus, OH 43210-1174

UNITED STATES

tran.350@osu.edu 


\title{
PACIFIC JOURNAL OF MATHEMATICS
}

\author{
msp.org/pjm
}

Founded in 1951 by E. F. Beckenbach (1906-1982) and F. Wolf (1904-1989)

\section{EDITORS}

Don Blasius (Managing Editor)

Department of Mathematics

University of California

Los Angeles, CA 90095-1555

blasius@math.ucla.edu

\author{
Paul Balmer \\ Department of Mathematics \\ University of California \\ Los Angeles, CA 90095-1555 \\ balmer@math.ucla.edu \\ Robert Finn \\ Department of Mathematics \\ Stanford University \\ Stanford, CA 94305-2125 \\ finn@math.stanford.edu \\ Sorin Popa \\ Department of Mathematics \\ University of California \\ Los Angeles, CA 90095-1555 \\ popa@math.ucla.edu
}

\author{
Vyjayanthi Chari \\ Department of Mathematics \\ University of California \\ Riverside, CA 92521-0135 \\ chari@math.ucr.edu \\ Kefeng Liu \\ Department of Mathematics \\ University of California \\ Los Angeles, CA 90095-1555 \\ liu@math.ucla.edu \\ Jie Qing \\ Department of Mathematics \\ University of California \\ Santa Cruz, CA 95064 \\ qing@ cats.ucsc.edu
}

\section{PRODUCTION}

Silvio Levy, Scientific Editor, production@msp.org

\section{SUPPORTING INSTITUTIONS}

ACADEMIA SINICA, TAIPEI

CALIFORNIA INST. OF TECHNOLOGY

INST. DE MATEMÁTICA PURA E APLICADA

KEIO UNIVERSITY

MATH. SCIENCES RESEARCH INSTITUTE

NEW MEXICO STATE UNIV.

OREGON STATE UNIV.

\author{
STANFORD UNIVERSITY \\ UNIV. OF BRITISH COLUMBIA \\ UNIV. OF CALIFORNIA, BERKELEY \\ UNIV. OF CALIFORNIA, DAVIS \\ UNIV. OF CALIFORNIA, LOS ANGELES \\ UNIV. OF CALIFORNIA, RIVERSIDE \\ UNIV. OF CALIFORNIA, SAN DIEGO \\ UNIV. OF CALIF., SANTA BARBARA
}

\author{
Daryl Cooper \\ Department of Mathematics \\ University of California \\ Santa Barbara, CA 93106-3080 \\ cooper@math.ucsb.edu \\ Jiang-Hua Lu \\ Department of Mathematics \\ The University of Hong Kong \\ Pokfulam Rd., Hong Kong \\ jhlu@maths.hku.hk \\ Paul Yang \\ Department of Mathematics \\ Princeton University \\ Princeton NJ 08544-1000 \\ yang@math.princeton.edu
}

These supporting institutions contribute to the cost of publication of this Journal, but they are not owners or publishers and have no responsibility for its contents or policies.

See inside back cover or msp.org/pjm for submission instructions.

The subscription price for 2014 is US $\$ 410 /$ year for the electronic version, and \$535/year for print and electronic.

Subscriptions, requests for back issues and changes of subscribers address should be sent to Pacific Journal of Mathematics, P.O. Box 4163, Berkeley, CA 94704-0163, U.S.A. The Pacific Journal of Mathematics is indexed by Mathematical Reviews, Zentralblatt MATH, PASCAL CNRS Index, Referativnyi Zhurnal, Current Mathematical Publications and Web of Knowledge (Science Citation Index).

The Pacific Journal of Mathematics (ISSN 0030-8730) at the University of California, c/o Department of Mathematics, 798 Evans Hall \#3840, Berkeley, CA 94720-3840, is published twelve times a year. Periodical rate postage paid at Berkeley, CA 94704, and additional mailing offices. POSTMASTER: send address changes to Pacific Journal of Mathematics, P.O. Box 4163, Berkeley, CA 94704-0163.

PJM peer review and production are managed by EditFLOW ${ }^{\circledR}$ from Mathematical Sciences Publishers.

\section{PUBLISHED BY}

\section{mathematical sciences publishers \\ nonprofit scientific publishing}

http://msp.org/

(C) 2014 Mathematical Sciences Publishers 


\section{PACIFIC JOURNAL OF MATHEMATICS}

Volume $269 \quad$ No. $2 \quad$ June 2014

Totaro's question for simply connected groups of low rank

257

JODI BLACK and RAMAN PARIMALA

Uniform hyperbolicity of the curve graphs

269

BRIAN H. BOWDITCH

Constant Gaussian curvature surfaces in the 3-sphere via loop groups

281

DAVID BRANDER, JUN-ICHI INOGUCHI and SHIMPEI KOBAYASHI

On embeddings into compactly generated groups

305

PIERRE-EMmanuel CAPRACE and Yves CORNULIER

Variational representations for $N$-cyclically monotone vector fields

ALFRED GALICHON and NASSIF GHOUSSOUB

Restricted successive minima

341

MaRTIN HenK and CARSTEN THIEL

Radial solutions of non-Archimedean pseudodifferential equations

355

ANATOLY N. KochubeI

A Jantzen sum formula for restricted Verma modules over affine Kac-Moody

algebras at the critical level

JOHANNES KÜBEL

Notes on the extension of the mean curvature flow

YAN LENG, ENTAO ZHAO and HaORAN ZHAO

Hypersurfaces with prescribed angle function

Henrique F. DE Lima, ERAldo A. Lima JR. and Ulisses L. Parente

Existence of nonparametric solutions for a capillary problem in warped products

JoRGE H. LiRA and GABRIELA A. WANDERLEY

A counterexample to the simple loop conjecture for $\operatorname{PSL}(2, \mathbb{R})$

425

KATHRYN MANN

Twisted Alexander polynomials of 2-bridge knots for parabolic representations

TAKAYUKI MORIFUJI and ANH T. TRAN

Schwarzian differential equations associated to Shimura curves of genus zero

FANG-TING TU

Polynomial invariants of Weyl groups for Kac-Moody groups 